\title{
Physicochemical Studies on Some Disaccharides (Sucrose, Lactose, Maltose) in Aqueous Media at 298.15 K
}

\author{
S. NITHIYANANTHAM* ${ }^{*}$ and L. PALANIAPPAN \\ *School of Physical Sciences and Nano Technology (Ultrasonics/Bio-Physics Divisions), \\ SRM University, Kattankulathur, Tamilnadu - 603 203, India \\ Department of Physics, Annamalai University, Annamalai Nagar, Chidambaram, \\ Tamilnadu - 602 008, India \\ s_nithu59@rediffmail.com
}

Received 16 June 2012 / Accepted 8 July 2012

\begin{abstract}
The ultrasonic velocity $(U)$, density $(\rho)$ and viscosity $(\eta)$ have been measured in the binary systems of some disaccharides (Sucrose, lactose and maltose) in aqueous media at 298.15 K. The acoustical parameters such as adiabatic compressibility $(\beta)$, free length $\left(L_{f}\right)$, free volume $\left(V_{f}\right)$, internal pressure $\left(\pi_{\mathrm{i}}\right)$, acoustical impedance $(\mathrm{Z})$, relative association $(\mathrm{Ra})$, Rao's constant (R) and Wada's constant (W) were calculated. The results are interpreted in terms of molecular interaction and structure variation between the components of the mixtures.
\end{abstract}

Keywords: Acoustical parameter, Thermodynamical parameter, Molecular interaction, Disaccharides, Ultrasonics

\section{Introduction}

In recent years ultrasonic investigation find extensive applications in probing in to the physico-chemical behaviour of binary liquid mixtures leading to an understanding of the liquid state $\mathrm{e}^{1-3}$. Ultrasonic technique has been adequately employed to investigate the properties of any substance to understand the nature of molecular interactions in pure liquid ${ }^{1}$ liquid mixtures ${ }^{2-5}$ and ionic interactions in electrolytic solutions ${ }^{6,7}$. Through the molecular interactions studies can be best carried out through spectroscopic methods ${ }^{8,9}$ the other non spectroscopic techniques such as dielectric ${ }^{10}$ magnetic $^{11}$ ultrasonic velocity and viscosity ${ }^{12}$ measurements have been widely used in field of interactions and structural aspect evaluations studies. The measurements of ultrasonic velocity have been adequately employed in understanding the nature of molecular systems and physicochemical behaviour in liquid mixtures ${ }^{13-16}$. 


\section{Experimental}

The ultrasonic velocity was measured using a single crystal variable path interferometer working at $2 \mathrm{MHz}$ by standard procedure. The accuracy of ultrasonic velocity determination in the solution is $\pm 0.001 \%$. The velocities were measured at temperature $298 \mathrm{~K}$. The density were measured using a corning specific gravity bottle by standard procedure and the viscosity was measured using Oswald's viscometer with an accuracy of $\pm 0.1 \%$.

\section{Results and Discussion}

The calculated parameters such as adiabatic compressibility $(\beta)$, inter molecular free length $\left(L_{f}\right)$, free volume $\left(V_{f}\right)$, internal pressure $\left(\pi_{i}\right)$ acoustic impedance $(Z)$, relative association $(R)$, Rao's constant (R) and Wada's constant (W) are calculated using the following relation (1- 8).

$$
\begin{aligned}
\beta & =1 / U^{2} \rho \\
L_{f} & =k_{T}(\beta)^{1 / 2} \\
V_{f} & =\left(M_{e f f} U / \eta k\right)^{3 / 2} \\
\pi_{i} & =b R T\left(\frac{K \eta}{U}\right)^{\frac{1}{2}}\left(\frac{\rho^{2 / 3}}{M_{\text {eff }}^{7 / 6}}\right) \\
Z & =U \rho \\
R_{A} & =\frac{\rho}{\rho_{o}}\left(\frac{U o}{U}\right)^{\frac{1}{3}} \\
R & =U^{\frac{1}{3}} V \\
W & =\beta^{1 / 7} V \quad \text { and }
\end{aligned}
$$

Table 1 lists the observed values for the three aqueous disaccharides viz. sucrose, lactose and maltose. In the present study all the three disaccharides are having the same molecular formula. However, sucrose (Figure 1) is having one unit of fructose (Figure .4) with glucose (Figure 5), lactose (Figure 2) having one unit of galactose (Figure 6) with glucose and for maltose (Figure 3), the two units of glucose, the units are held combined by an oxygen atom.

Table 1. Measured values such as ultrasonic velocity (U), density $(\rho)$ and viscosity $(\eta)$ of various molariy (m) of (sucrose, lactose and maltose) in water at $298.15 \mathrm{~K}$

\begin{tabular}{cccccccccc}
\hline & \multicolumn{3}{c}{$\begin{array}{c}\mathrm{U} \\
\mathrm{m}\end{array}$} & \multicolumn{3}{c}{$\mathrm{ms}^{-1}$} & \multicolumn{3}{c}{$\begin{array}{c}\rho \\
\mathrm{kgm}^{-3}\end{array}$} \\
\cline { 2 - 10 } & Sucrose & Lactose & Maltose & Sucrose & Lactose & Maltose & Sucrose Lactose Maltose \\
\hline 0.01 & 1503.6 & 1502.5 & 1503.0 & 1001.5 & 998.6 & 1003.5 & 0.898 & 0.900 & 0.900 \\
0.02 & 1505.2 & 1503.9 & 1504.6 & 1002.4 & 999.7 & 1004.6 & 0.912 & 0.908 & 0.908 \\
0.03 & 1507.0 & 1505.6 & 1505.8 & 1003.2 & 1000.5 & 1005.4 & 0.926 & 0.917 & 0.918 \\
0.04 & 1509.3 & 1507.8 & 1507.1 & 1004.7 & 1001.4 & 1006.4 & 0.940 & 0.927 & 0.927 \\
0.05 & 1511.0 & 1509.1 & 1508.7 & 1005.2 & 1002.2 & 1007.3 & 0.956 & 0.941 & 0.936 \\
0.06 & 1512.4 & 1511.5 & 1510.5 & 1006.0 & 1004.6 & 1008.1 & 0.968 & 0.954 & 0.946 \\
0.07 & 1513.5 & 1512.8 & 1512.8 & 1007.1 & 1006.1 & 1009.2 & 0.975 & 0.971 & 0.964 \\
0.08 & 1515.2 & 1514.5 & 1514.9 & 1008.7 & 1007.6 & 1010.4 & 0.982 & 1.000 & 0.978 \\
0.09 & 1516.8 & 1516.2 & 1516.7 & 1009.6 & 1009.6 & 1011.4 & 0.991 & 1.007 & 0.996 \\
0.10 & 1519.0 & 1517.3 & 1518.8 & 1010.3 & 1010.5 & 1012.6 & 1.016 & 1.027 & 1.019 \\
\hline
\end{tabular}




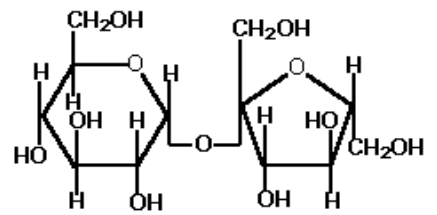

Figure 1. Structure of sucrose

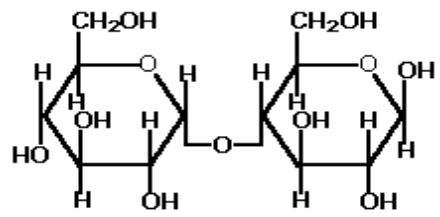

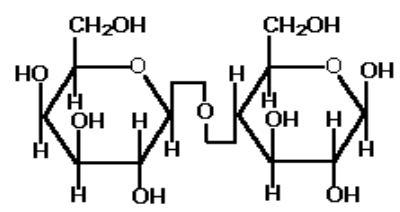

Figure 2. Structure of lactose

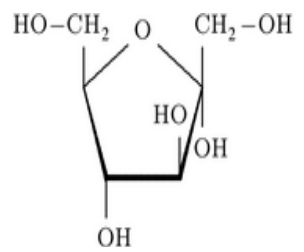

Figure 4. Structure of $D$-frucctose

Figure 3. Structure of maltose<smiles>O=CC(O)C(O)C(O)CO</smiles>

Figure 5. Structure of $D$-glucpse<smiles>CC(O)(CO)C(O)C(O)C(O)C=O</smiles><smiles>OC[C@H]1C[C@@H](O)[C@H](O)[C@H]1O</smiles>

D-Galactose haworths

\section{D-Galactose fishers}

Figure 6. Structure of galactose

Referring the Table 1, it suggests that the value of sound velocity and their variation with molarity follows almost the same trend for all disaccharides taken up for the present work. It is clear that for any given molarity, sucrose shows a maximum sound velocity, whereas for lactose, it is minimum. As regards maltose and lactose, the maltose recorded the higher velocity from 0.01 to 0.03 and 0.07 to 0.10 and in remaining molarities maltose recorded the lower value of velocity. But considering the variation in density, minimum density is observed for lactose, whereas for maltose maximum values are recorded. The observed density values are minimum for lactose upto $0.09 \mathrm{~m}$, but in $0.10 \mathrm{~m}$, the least value is shifted to sucrose.

The observed viscosity values are maximum for lactose at $0.01 \mathrm{~m}$ and from $0.08 \mathrm{~m}$ to $0.10 \mathrm{~m}$, but in the range of $0.02 \mathrm{~m}$ to $0.07 \mathrm{~m}$, the maximum values are shifted to sucrose. The minimum value of $\eta$ for sucrose at $0.01 \mathrm{~m}$ and 0.07 to $0.1 \mathrm{~m}$, but in the range of 0.02 and $0.03 \mathrm{~m}$ the least values are shifted to lactose and then it shifted to $0.08 \mathrm{~m}$ to $0.09 \mathrm{~m}$ for maltose. The co-efficient of viscosity of liquid have some special property that determines the inner nature of the liquid medium. 
Sucrose is formed by the elimination of a molecule of water from the combination of glycosidic hydroxyl groups of $\alpha$ - $D$-glucose and $\beta$-D-glucose. The fructose possess the furonose ring structure in the sucrose molecule, the pyranose ring is the dominant and more stable form in the free ketohexose. For sucrose formation both the carbonyl groups of individual units are involved in the formation of glycosidic bond. Sucrose contains no active group and so it does not exhibit mutarotation ${ }^{17}$. This is a non reducing sugar. Free carbonyl is the active group of lactose and hemiacetyl is an active group of maltose.

The analysis towards the type and nature of existing interactions are done with the same thermoacoustical parameters as stated before in monosaccharides. From the analysis the structural variations can be analysed. Compressibility values are higher for loosely packed molecules and lower for strong packing and have a maximum interaction. The smooth decrease in adiabatic compressibility with respect to increase in molarity suggests that the medium become very less compressible. The Table 2 shows the derived parameters for sucrose, lactose and maltose respectively. Referring the Table 2, it is evident that the adiabatic compressibility is a decreasing trend with increasing molarity of all disaccharides in general.

Table 2. Calculated values such as adiabatic compressibility $(\beta)$, freelength $\left(L_{f}\right)$, freevolume $\left(V_{f}\right)$, internal pressure $\left(\pi_{\mathrm{i}}\right)$, acoustical impedance $(\mathrm{Z})$, relative association $\left(\mathrm{R}_{\mathrm{A}}\right)$, Rao's constant (R) and Wada's constant (W) for various molarity (m) of (sucrose, lactose, maltose in) water at $298.15 \mathrm{~K}$

\begin{tabular}{|c|c|c|c|c|c|c|c|c|}
\hline $\mathrm{m}$ & $\begin{array}{c}\beta \times 10^{10} \\
\mathrm{~N}^{-1} \mathrm{~m}^{2}\end{array}$ & $\begin{array}{c}\mathrm{L}_{\mathrm{f}} \times 10^{11} \\
\mathrm{~m}\end{array}$ & $\begin{array}{l}\mathrm{V}_{\mathrm{f}} \times 10^{8} \\
\mathrm{~m}^{3} \mathrm{~mol}^{-1}\end{array}$ & $\begin{array}{c}\pi_{\mathrm{i}} \times 10^{-9} \\
\mathrm{Nm}^{-2}\end{array}$ & $\begin{array}{l}\mathrm{Z} \times 10^{-6} \\
\mathrm{kgm}^{-2} \mathrm{~s}^{-1}\end{array}$ & $\mathrm{R}_{\mathrm{A}}$ & $\begin{array}{c}\mathrm{R} \times 10^{1} \\
\mathrm{~m}^{10 / 3} \\
\mathrm{~s}^{-1 / 3} \mathrm{~mol}^{-1}\end{array}$ & $\begin{array}{l}\mathrm{W} \times 10^{1} \\
\mathrm{~m}^{3} \mathrm{~mol}^{-1} \\
\left(\mathrm{~N} / \mathrm{m}^{2}\right)^{1 / 7}\end{array}$ \\
\hline \multicolumn{9}{|c|}{ Sucrose + water } \\
\hline 0.01 & 4.416 & 4.193 & 1.877 & 2.711 & 1.506 & 1.0030 & 2.0656 & 3.9122 \\
\hline 0.02 & 4.403 & 4.187 & 1.846 & 2.723 & 1.509 & 1.0036 & 2.0711 & 3.9230 \\
\hline 0.03 & 4.389 & 4.180 & 1.818 & 2.732 & 1.512 & 1.0040 & 2.0770 & 3.9343 \\
\hline 0.04 & 4.370 & 4.171 & 1.790 & 2.743 & 1.516 & 1.0048 & 2.0820 & 3.9442 \\
\hline 0.05 & 4.357 & 4.165 & 1.755 & 2.756 & 1.519 & 1.0050 & 2.0881 & 3.9560 \\
\hline 0.06 & 4.346 & 4.159 & 1.735 & 2.762 & 1.522 & 1.0055 & 2.0937 & 3.9669 \\
\hline 0.07 & 4.335 & 4.154 & 1.726 & 2.763 & 1.524 & 1.0064 & 2.0986 & 3.9766 \\
\hline 0.08 & 4.318 & 4.146 & 1.718 & 2.765 & 1.528 & 1.0076 & 2.1026 & 3.9849 \\
\hline 0.09 & 4.305 & 4.140 & 1.705 & 2.767 & 1.531 & 1.0082 & 2.1081 & 3.9956 \\
\hline 0.10 & 4.295 & 4.135 & 1.765 & 2.792 & 1.535 & 1.0086 & 2.1138 & 4.0067 \\
\hline \multicolumn{9}{|c|}{ Lactose + water } \\
\hline 0.01 & 4.436 & 4.202 & 1.868 & 2.710 & 1.500 & 1.0003 & 2.0715 & 3.9220 \\
\hline 0.02 & 4.423 & 4.196 & 1.858 & 2.711 & 1.504 & 1.0011 & 2.0769 & 3.9326 \\
\hline 0.03 & 4.409 & 4.190 & 1.843 & 2.714 & 1.506 & 1.0015 & 2.0831 & 3.9445 \\
\hline 0.04 & 4.392 & 4.182 & 1.826 & 2.718 & 1.510 & 1.0021 & 2.0893 & 3.9564 \\
\hline 0.05 & 4.381 & 4.176 & 1.798 & 2.727 & 1.512 & 1.0025 & 2.0952 & 3.9680 \\
\hline 0.06 & 4.357 & 4.165 & 1.773 & 2.738 & 1.518 & 1.0043 & 2.0985 & 3.9752 \\
\hline 0.07 & 4.343 & 4.158 & 1.738 & 2.753 & 1.522 & 1.0055 & 2.1029 & 3.9844 \\
\hline 0.08 & 4.327 & 4.150 & 1.673 & 2.785 & 1.526 & 1.0066 & 2.1076 & 3.9938 \\
\hline 0.09 & 4.308 & 4.142 & 1.667 & 2.786 & 1.531 & 1.0083 & 2.1111 & 4.0014 \\
\hline 0.10 & 4.299 & 4.137 & 1.628 & 2.803 & 1.533 & 1.0089 & 2.1169 & 4.0128 \\
\hline
\end{tabular}




\begin{tabular}{lllllllll}
\hline & \multicolumn{7}{c}{ Maltose + water } \\
0.01 & 4.411 & 4.191 & 1.870 & 2.718 & 1.508 & 1.0051 & 2.0618 & 3.9061 \\
0.02 & 4.397 & 4.184 & 1.858 & 2.720 & 1.512 & 1.0058 & 2.0672 & 3.9169 \\
0.03 & 4.387 & 4.179 & 1.838 & 2.724 & 1.514 & 1.0064 & 2.0731 & 3.9283 \\
0.04 & 4.375 & 4.173 & 1.825 & 2.727 & 1.517 & 1.0071 & 2.0787 & 3.9392 \\
0.05 & 4.361 & 4.167 & 1.809 & 2.731 & 1.520 & 1.0076 & 2.0845 & 3.9506 \\
0.06 & 4.348 & 4.160 & 1.792 & 2.734 & 1.523 & 1.0080 & 2.0907 & 3.9625 \\
0.07 & 4.330 & 4.152 & 1.757 & 2.749 & 1.527 & 1.0086 & 2.0964 & 3.9738 \\
0.08 & 4.312 & 4.144 & 1.731 & 2.759 & 1.531 & 1.0094 & 2.1018 & 3.9845 \\
0.09 & 4.298 & 4.136 & 1.697 & 2.772 & 1.534 & 1.0100 & 2.1076 & 3.9956 \\
0.10 & 4.281 & 4.128 & 1.650 & 2.794 & 1.538 & 1.0107 & 2.1130 & 4.0063 \\
\hline
\end{tabular}

The intermolecular free length $\left(\mathrm{L}_{\mathrm{f}}\right)$ is an another important factor for determining the interactions between the components of the solution. Free lengths reflect the same trend as that of adiabatic compressibility. The medium in each solution has only one solute and their structure depends upon the glycosyl bond and the type of active group.

As regards lactose solution, the $\beta$ and $L_{f}$ values are maximum, that conveys more available free space between the molecules than compared with sucrose and maltose. It may be taken as a consequence of lesser molecular size. As lactose exist in three different forms, lesser molecular size can be attributed only for $\alpha$-lactose and this is the predominating structure of lactose. However, as molarity of lactose increases $\beta$ and $\mathrm{L}_{\mathrm{f}}$ values are in decreasing trend. This is possible only if the structure of $\alpha$-lactose may be changed into any one of other forms.

The Table 2 also reveals that the $V_{\mathrm{f}}$ values are maximum in $0.01 \mathrm{~m}, 0.09 \mathrm{~m}$ and $0.10 \mathrm{~m}$ for sucrose and maximum $\mathrm{V}_{\mathrm{f}}$ values shifted to $0.02 \mathrm{~m}$ to $0.05 \mathrm{~m}$ for lactose and further shifted to $0.06 \mathrm{~m}$ to $0.08 \mathrm{~m}$ for maltose whereas, the minimum values observed in $0.01 \mathrm{~m}$, $0.08 \mathrm{~m}$ to $0.10 \mathrm{~m}$ for lactose and $0.02 \mathrm{~m}$ to $0.05 \mathrm{~m}$ it is shifted to sucrose.

The internal pressure is the cohesive force, which is the resultant of force of attraction and force of repulsion between the molecules. The same tables further reveals that the internal pressure $\left(\pi_{\mathrm{i}}\right)$ is maximum in $0.01 \mathrm{~m}$ for maltose, $0.02 \mathrm{~m}$ to $0.08 \mathrm{~m}$ for sucrose and then shifted to $0.09 \mathrm{~m}$ and $0.10 \mathrm{~m}$ for lactose, whereas the minimum $\pi_{\mathrm{i}}$ values are observed in $0.01 \mathrm{~m}$ to $0.05 \mathrm{~m}$ and $0.08 \mathrm{~m}$ for lactose, $0.06 \mathrm{~m}$ and $0.07 \mathrm{~m}$ for maltose and further shifted to $0.09 \mathrm{~m}$ and $0.10 \mathrm{~m}$ for sucrose $\mathrm{e}^{18,19}$.

As already stated, the structural variations with rearrangements of molecule with in the solution, in addition to the presence of active group, may be attributed for the observed changes. No more active group in case of sucrose, whereas in case of lactose and maltose, free carbonyl or free hemiacetyl active group exist along with the possibility of all the three forms at all but at various proportions and hence the extent of these structure leads to their irregular variations in the solutions of lactose and maltose whereas no such variation appeared in sucrose solution.

The observed trend of acoustical impedance $(\mathrm{Z})$ and relative association $\left(\mathrm{R}_{\mathrm{A}}\right)$ for each of disaccharides with molarity is in general increases. This indicates that the possibility of solutesolute interaction and chances for enhancing the complexation are more in the solution under study. Referring the same Table 2 and comparing the values of $Z$ and $R_{A}$, it is observed that $Z$ values are maximum for maltose and least for lactose. Considering $R_{A}$ values, it is maximum for maltose and minimum for lactose up to $0.08 \mathrm{~m}$ and 0.09 to $0.10 \mathrm{~m}$ sucrose shows minimum.

The maximum $\mathrm{R}_{\mathrm{A}}$ of maltose is due to the availability of hemiacetyl group, where free sites are more available. Further, the vacant sites are also responsible for the observed 
Chem Sci Trans., 2013, 2(1), 35-40

increasing trend of these $\mathrm{Z}$ and $\mathrm{R}_{\mathrm{A}}$ values. It is evident that among the disaccharides taken up for the present study, maltose exhibit maximum interaction that depends on the type of active group and molarity, however the type is of solute-solute interaction.

The molar sound velocity (R) and their compressibility (W) for all disaccharides are in increasing trend with the molarity. This indicates the magnitude of interactions is enhanced. This increasing trend of $\mathrm{R}$ and $\mathrm{W}$ indicates that availability of more number of components in a given region of space. This leads to a tight packing of the medium and thereby increase the interactions. On comparing the values observed for all the disaccharides, lactose records maximum, whereas maltose records minimum values. This may be attributed to the fact that lactose possesses three different structures and further the velocity in the parameters that depends more on the number of components than the geometry density, lactose appears to possess maximum interaction as that of sucrose $\mathrm{e}^{20,21}$.

\section{Conclusion}

1. All the disaccharides show an increasing trend of sound velocity with molarity.

2. The presence of solute-solute interaction is confirmed.

3. The strength of interaction is found to be molarity sensitive of the disaccharide.

4. Among the three disaccharides, maltose shows maximum solute-solvent type interaction whereas lactose maximum solute-solute interactions.

\section{References}

1. Sheshagiri Rao M G, Indian J Pure Appl Phys., 1971, 9, 169.

2. Sheshagiri K and Reddy K C, Acustica (Germany), 1973, 29, 59-65.

3. Ramamoorthy K and Varadachari P S, Indian J Pure Appl phys., 1973, 11, 238-2442.

4. $\quad$ Kaulgud MV and Patil K J, Indian J Pure Appl Phys., 1975, 13, 322-324.

5. $\quad$ Tiwari V and Pandey J D, Indian J Pure Appl Phys., 1980, 18, 51-57.

6. Satya Narayanamurthy M, Curr Sci., 1964, 33, 364.

7. $\quad$ Soitkar V S and Jajoo S N, Acoust Lett., 1984, 7(12), 1991-1998.

8. Suryanarayana C V, J Acoust Soc India, 1983, 13, XI.

9. $\quad$ Fletcher A, J Phys Chem., 1969, 73, 2217-2225.

10. Pimental G C and Maclellan A L, The Hydrogen bond, (WH Freeman and Co, san Fransisco), 1960.

11. Hobbs M E and Bates W W, J Am Chem Soc., 1952, 74, 746.

12. $\quad$ Lin W and Tsay S J, J Phys Chem., 1970, 74, 1037.

13. Manisha Gupta and Shukla J P, Indian J Pure Appl Phys., 1996, 34, 772.

14. Pandey J D, Rai R D, Shukla A k and Misra N, Indian J Pure Appl Phys., 1993, 31, 84.

15. Pankaj and Sharma C, Ultrasonics, 1991, 29, 344-347.

16. Velmourougane S, Nambinarayanan T K, Srinivasa Rao A and Krishnan B, Indian J Phys., 1987, 61B, 105-109.

17. Chakrabarti B K, Ghosh H N and Sahana S N, Modern Human Physiology, The New Book Stall, Calcutta (India), $1^{\text {st }}$ Edition, 1972.

18. Nithiyanantham S and Palaniappan L, Euro Phy J Appl Phys., 2011, 3, 31101.

19. Nithiyanantham S and Palaniappan L, Fron Chem Chi., 2011, 6(2), 147.

20. Vanathi V, Mullainathan S and Nithiyanantham S, Rus J Phy Chem A, 2012, 86(8), 1204.

21. Vanathi V, Mullainathan S and Nithiyanantham S, E-J Chem., 2012. 